Systematic Review

\title{
Is Traumatic and Non-Traumatic Neck Pain Associated with Brain Alterations? - A Systematic Review
}

Robby De Pauw, MSc ${ }^{1}$, Iris Coppieters, MSc ${ }^{1,2}$, Mira Meeus, $\mathrm{PhD}^{1,2,3}$,

Karen Caeyenberghs, $\mathrm{PhD}^{4}$, Lieven Danneels, $\mathrm{PhD}^{1}$, and Barbara Cagnie, $\mathrm{PhD}^{1}$

From: ${ }^{1}$ Department of Rehabilitation Sciences and

Physiotherapy, Faculty of Medicine and Health Sciences, Ghent University, Belgium; ${ }^{2}$ Pain in Motion international research group, www.paininmotion.be; ${ }^{3}$ Department of Rehabilitation

Sciences and Physiotherapy, Faculty of Medicine and Health Sciences, University of Antwerp Belgium; ${ }^{4}$ School of Psychology,

Faculty of Health Sciences,

Australian Catholic University,

Australia

Address Correspondence: Robby De Pauw, MSc Department of Rehabilitation Sciences and Physiotherapy, Faculty of Medicine and Health

Sciences, Ghent University, St. Pietersnieuwstraat 33, 9000 Gent,

Belgium

Email: robby.depauw@ugent.be

Disclaimer: De Pauw and Dr. Coppieters contributed equally to this work. There was no external funding in the preparation of this manuscript.

Conflict of interest: Each author certifies that he or she, or a member of his or her immediate

family, has no commercial association (i.e., consultancies, stock ownership, equity interest, patent/licensing arrangements, etc.) that might pose a conflict of interest in connection with the

submitted manuscript.

Manuscript received: o8-22-2016

Revised manuscript received: 11-14-2016

Accepted for publication: 11-21-2016

Free full manuscript: www.painphysicianjournal.com
Background: Chronic neck pain affects $50 \%-85 \%$ of people who have experienced an acute episode. This transition and the persistence of chronic complaints are believed to be mediated by brain alterations among different central mechanisms.

Objectives: This study aimed to systematically review and critically appraise the current existing evidence regarding structural and functional brain alterations in patients with whiplash associated disorders (WAD) and idiopathic neck pain (INP). Additionally, associations between brain alterations and clinical symptoms reported in neck pain patients were evaluated.

Study Design: Systematic review.

Methods: The present systematic review was performed according to the PRISMA guidelines. PubMed, Web of Science, and Cochrane databases were searched. First, the obtained articles were screened based on title and abstract. Secondly, the screening was based on the full text. Risk of bias in included studies was investigated.

Results: Twelve studies met the inclusion criteria. Alterations in brain morphology and function, including perfusion, neurotransmission, and blood oxygenation level dependent-signal, were demonstrated in chronic neck pain patients. There is some to moderate evidence for both structural and functional brain alterations in patients with chronic neck pain. In contrast, no evidence for structural brain alterations in acute neck pain patients was found.

Limitations: Only 12 articles were included, which allows only cautious conclusions to be drawn.

Conclusion: Brain alterations were observed in both patients with chronic WAD and chronic INP. Furthermore, more evidence exists for brain alterations in chronic WAD, and different underlying mechanisms might be present in both pathologies. In addition, pain and disability were correlated with the observed brain alterations. Accordingly, morphological and functional brain alterations should be further investigated in patients with chronic WAD and chronic INP with newer and more sensitive techniques, and associative clinical measurements seem indispensable in future research.

Key words: Traumatic neck pain, idiopathic non-traumatic neck pain, brain alterations, magnetic resonance imaging, single photon emission computed tomography, photon emission tomography, chronic neck pain

Pain Physician 2017; 20:245-260 


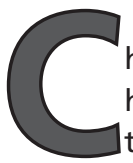
hronic neck pain affects many people who have encountered an episode of neck pain throughout their lifetime with $50 \%-85 \%$ of people who experienced neck pain reporting neck pain $1-5$ years later $(1,2)$.

Two groups of non-specific neck pain patients have been identified in the literature, i.e., patients who have developed neck pain after a traumatic event, which are referred to as patients with whiplash associated disorders (WAD) (3), and patients with non-traumatic idiopathic neck pain (INP). Whiplash injuries usually result from rear-end motor vehicle collisions resulting in acceleration-deceleration mechanisms of forces acting on the neck and the head (4). The traumatic neck pain group is frequently seen as a special case, as these patients more often develop chronic complaints $(5,6)$, which do not only consist of neck pain and/or headaches, but also include other symptoms such as dizziness (7), motor dysfunction (8-13), disturbed central pain processing or central sensitization $(14,15)$, and cognitive impairment $(5,16-19)$; hence the term "associated disorders." In addition, patients with INP have developed neck pain without any clear underlying cause (20). These patients are mostly not characterized by central sensitization (21), cognitive impairment, and dizziness; however, they also display motor dysfunctions $(22,23)$ and chronic or recurrent pain.

The cause of this diversity in symptoms observed in patients suffering from acute and chronic pain is still not entirely clear. Some have suggested that alteration of the central nervous system could explain this diversity $(22,24-27)$, and a theoretical framework for central nervous system alterations, such as brain alterations, has already been constructed for acute and chronic pain $(28,29)$. Surprisingly, only a few have tried to analyze and publish results that might support these theories. In addition, it is known that a trauma can result in mild traumatic brain injury (MTBI) (30), which is associated with clinical symptoms similar to these observed in patients with WAD. However, information on the impact of a whiplash trauma on the brain remains scarce in patients with neck pain. To answer the question if brain alterations play a role in patients suffering from chronic pain, the application of new brain analysis tools is rising (31-36). However, only limited research is available on alterations in brain morphology and function in patients with WAD and INP.

Brain alterations are often categorized into functional alterations and morphological or structural alterations. Brain function, which reflects the amount of activity that the brain generates at a certain location, is often measured via its blood perfusion and/or metabolism. Single photon emission computed tomography (SPECT) and photon emission tomography (PET) are both applied for this purpose (36). Both methods use radiopharmaceutical tracers to assess the brain's perfusion and/or metabolism. Active brain regions have a higher need of oxygen and glucose, which is reflected in a higher perfusion and/or metabolism $(37,38)$. These changes in metabolite concentration are captured and reflected in the image signal intensity. Another method to analyze brain function is by applying functional magnetic resonance imaging (MRI). The MRI method most often used to provide information related to brain function is called blood oxygenation level dependent (BOLD) contrast imaging (39). This method is based on MRI made sensitive to changes in oxygen consumption with an increase in consumption reflecting higher signal intensities in these BOLD images.

Besides brain function, brain morphology is also believed to be altered in certain pain conditions $(40,41)$. MRI has achieved the level of gold standard for measuring brain morphology, typically through voxel- or surface-based methods (42). Both methods provide information on white and grey matter volume.

According to our knowledge, no systematic review has critically summarized the current evidence regarding brain alterations in patients with WAD and patients with INP. This systematic review determines the present state of the art and steers further research in patients with WAD and INP. The aim of this systematic review is to review and critically appraise the current existing evidence related to structural and functional brain alterations in patients with WAD and INP. In addition, this review evaluates the association between these brain alterations and the different clinical symptoms reported in patients with neck pain.

\section{Methods}

\section{Protocol}

This systematic review applies the guidelines issued in the PRISMA statement, an adaptation of the QUORUM statement for reporting systematic reviews $(43,44)$.

\section{Information Sources}

The electronic databases PubMed (http://www. ncbi.nlm.nih.gov/pubmed), Cochrane Library (www. cochranelibrary.com), and Web of Science (www. webofscience.com) were searched to identify relevant 
articles. Additionally, to make the search as complete as possible, reference lists of the eligible papers were screened. Databases were searched on December 4, 2015, and all articles were screened afterwards on eligibility criteria.

\section{Literature Search Strategy}

The search strategy was based on a combination of Mesh-terms (only for searching PubMed) and free text words derived from the following PICO format: participants $(P)$ had to suffer from acute, subacute, or chronic INP, neck injuries, or WAD; the measurement instrument (I) had to include medical brain imaging techniques such as MRI, PET, and SPECT; and the outcome (O) had to refer to brain alterations including brain function and brain morphology. The complete entered search strategy in PubMed was ("neck pain" OR "Neck Pain"[Mesh] OR "whiplash" OR "Whiplash Injuries"[Mesh] OR "neck injury" OR "Neck Injuries"[Mesh]) AND ("brain imaging" OR "Neuroimaging"[Mesh]OR "fMRI" OR "rs-fMRI" OR "MRI" OR "Magnetic Resonance Imaging"[Mesh] OR "Magnetic Resonance Imaging" OR "Positron Emission Topography" $O R$ "PET" OR "Positron-Emission Tomography"[Mesh] OR "CT" OR "Computed Tomography" OR "SPECT" OR "Tomography, X-Ray Computed"[Mesh]) AND ("brain morphology" OR "Brain"[Mesh] OR "brain" OR "white matter" OR "grey matter" OR "gray matter" OR "White Matter"[Mesh] OR "Gray Matter"[Mesh] OR "brain function" OR "resting state" OR "BOLD" OR "brain volume").

\section{Eligibility Criteria}

Only patient-controlled, cross-sectional, and cohort studies reporting on brain alterations in non-specific non-traumatic and traumatic acute, subacute, or chronic neck pain patients were eligible for inclusion in this systematic review. No restrictions on publication date or status were imposed. Studies had to be written in English, Dutch, or French to be included in this systematic review. Adult participants, 18 or older, suffering from idiopathic neck pain or a whiplash injury were considered eligible. Assessment of brain alterations should focus on brain structure/morphology and/or brain function, and only imaging techniques, such as SPECT, PET, and MRI were included in this systematic literature review.

\section{Study Selection}

Two reviewers, BC and RDP, independently screened all articles on eligibility in a standardized manner. Disagreement between the reviewers was resolved by consensus.

\section{Data Collection Process and Items}

Data were extracted from eligible papers in a standardized manner by RDP, and the extracted data were checked afterwards by a second reviewer, IC, who made changes where necessary. Disagreements were resolved by consensus or the opinion of a third reviewer, MM. Extracted data consisted of author and year of publication, description of the included population and, if available, the controls (sample size, neck pain type, mean age, gender, duration of complaints before scanning), the imaging protocol (SPECT, PET, or MRI) with technical information on the scanning sequence or radiopharmaceutical tracers used in the scanning procedure, the brain tissue class and areas that were investigated during the scanning sequence, and the main findings and associations with clinical measures (Spearman correlations $\left[r_{s}\right]$, Pearson correlations $\left[r_{p}\right]$ ).

\section{Risk of Bias in Individual Studies}

To assess the methodological quality of all eligible papers, 2 independent reviewers, RDP and IC, both $\mathrm{PhD}$ candidates experienced in conducting systematic reviews, screened all articles on risk of bias using a modified version of The Newcastle-Ottawa Scale (NOS) for assessing the quality of non-randomized studies in metaanalyses (www.ohri.ca/programs/clinical_epidemiology/ oxford.asp) (45). This checklist is recommended for casecontrol and cohort studies (46) and has been proposed by the Cochrane Collaboration (www.cochrane.org). Two different checklists were used to assess the quality of cross-sectional case-control and cohort studies. The case-control checklist evaluates selection of the population (case definition, representativeness of cases, and selection of controls) and comparability (controlled for the most important confounders such as age, gender, education level, and BMI). The cohort checklist evaluates selection (representativeness of the cohort, selection controls, case definition, new cases), comparability (controlled for the most important confounders [age, gender, education level, BMI]), and exposure (followup). In addition, for both checklists, 4 scoring items specifically developed for the content of this systematic review were added (description of MRI protocol, quality control of images, blindness of researcher, same method applied for cases and controls). Further details on the different criteria are displayed in Table 1. Each cross-sectional study could reach a maximum score of 9 and each cohort study could reach a maximum score of 11 on the modified NOS, representing the highest methodological quality. 
Table 1. Methodological quality of included studies.

\begin{tabular}{|c|c|c|c|c|c|c|c|c|c|}
\hline \multicolumn{10}{|c|}{ Cross-sectional Study Design } \\
\hline & $\begin{array}{c}\text { Case } \\
\text { Definition }\end{array}$ & $\begin{array}{c}\text { Case } \\
\text { Description }^{2}\end{array}$ & $\begin{array}{l}\text { Selection } \\
\text { Controls }^{3}\end{array}$ & Comparability $^{4}$ & MRI $^{5 a}$ & \begin{tabular}{|c|} 
Quality \\
Control $^{5 \mathbf{b}}$
\end{tabular} & Blindness $^{5 c}$ & $\begin{array}{c}\text { Same } \\
\text { Method }^{6}\end{array}$ & $\begin{array}{c}\text { Total /9 } \\
(\%)\end{array}$ \\
\hline $\begin{array}{l}\text { Borchgrevink et al } \\
\text { (1997) (47) }\end{array}$ & + & + & + & + & - & - & + & + & $6(67)$ \\
\hline $\begin{array}{l}\text { Sundström et al } \\
(2006)(55)\end{array}$ & + & - & ++ & + & + & - & - & - & $5(55)$ \\
\hline $\begin{array}{l}\text { Linnman et al } \\
\text { (2009) (54) }\end{array}$ & + & - & - & + & + & + & - & + & $5(55)$ \\
\hline $\begin{array}{l}\text { Sturzenegger et al } \\
(2008)(50)\end{array}$ & + & + & ++ & + & + & - & + & + & $8(89)$ \\
\hline $\begin{array}{l}\text { Radanov et al } \\
\text { (1999) (58) }\end{array}$ & + & - & - & - & + & - & - & + & $3(25)$ \\
\hline $\begin{array}{l}\text { Otte et al } \\
(1997)(56)\end{array}$ & - & - & - & - & + & - & - & + & $2(22)$ \\
\hline $\begin{array}{l}\text { Lorberboym et al } \\
\text { (2002) (57) }\end{array}$ & + & - & - & - & + & + & + & - & $4(44)$ \\
\hline $\begin{array}{l}\text { Linnman et al } \\
(2010)(52)\end{array}$ & + & + & ++ & + & + & + & - & + & $8(89)$ \\
\hline $\begin{array}{l}\text { Freitag et al } \\
(2001)(51)\end{array}$ & + & - & + & - & + & + & + & + & $6(67)$ \\
\hline $\begin{array}{l}\text { Bakhtadze et al } \\
(2012)(53)\end{array}$ & + & + & - & - & + & - & - & - & $3(25)$ \\
\hline
\end{tabular}

$+=$ score fulfilled; - = score not fulfilled

Modified Newcastle-Ottawa Quality Assessment Scale: cross-sectional studies: 1 = Is the case definition adequate? (Independent validation or self-reported); 2 = Representativeness of cases (Random sample: description of area, hospital and clinic); 3 = Selection of controls (Community controls with no history of disease (++), Hospital controls with no history of disease (+)); 4 = Comparability (Controlled for the most important confounders [age, gender, cognition, BMI]); $5 \mathrm{a}=$ Description of MRI protocol (full description and optimal sequencing protocol); $5 \mathrm{~b}=\mathrm{Quality}$ control of images (fully described); $5 \mathrm{c}=$ Blindness (researchers were blinded for patient's status); $5 \mathrm{~d}=$ Same method used for controls/cases (yes)

\begin{tabular}{|c|c|c|c|c|c|c|c|c|c|c|c|}
\hline \multicolumn{12}{|c|}{ Cohort Study Design } \\
\hline & $\begin{array}{l}\text { Exposed } \\
\text { Cohort }^{1}\end{array}$ & $\begin{array}{l}\text { Selection } \\
\text { Controls }^{2}\end{array}$ & $\begin{array}{c}\text { Ascertainment } \\
\text { of Exposure }\end{array}$ & $\begin{array}{c}\text { New } \\
\text { Cases }^{4}\end{array}$ & Parity $^{5}$ & MRI $^{\mathbf{a}}$ & $\begin{array}{c}\text { Quality } \\
\text { Control }^{6 b}\end{array}$ & Blindness $^{6 c}$ & $\begin{array}{c}\text { Same } \\
\text { Method }^{7}\end{array}$ & $\begin{array}{c}\text { Follow- } \\
\text { up }^{8}\end{array}$ & $\begin{array}{c}\text { Total } \\
/ 11 \\
(\%) \\
\end{array}$ \\
\hline $\begin{array}{l}\text { Karlsborg } \\
\text { et al (1998) } \\
(48)\end{array}$ & - & - & + & - & - & - & - & - & - & ++ & $3(27)$ \\
\hline $\begin{array}{l}\text { Obermann } \\
\text { et al } \\
(2009) \\
(49)\end{array}$ & - & - & + & - & + & + & - & - & + & ++ & $6(54)$ \\
\hline
\end{tabular}

$+=$ score fulfilled; $-=$ score not fulfilled

$1=$ Representativeness of exposed cohort (truly representative average in the community); $2=$ Selection controls (Drawn from the same community); 3 = Ascertainment of exposure (Independent validation or self-reported); $4=$ New cases (Yes); $5=$ Comparability (Controlled for the most important confounders [age, sex, cognition, $\mathrm{BMI}$ ); $6 \mathrm{a}=$ Description of MRI protocol (Full description and optimal sequencing protocol); $6 \mathrm{~b}=$ Quality control of images (fully described); $6 c=$ Blindness (researcher was blinded for patient's status); 7 = Same method used for controls/cases (yes); $8=$ Follow-up (Long enough $[>3$ months] and $>80 \%[++],>80 \%[+]$ )

Based on the study design and risk of bias, a level of evidence was given to every study, according to the 2005 classification system of the Dutch Institute for
Healthcare Improvement CBO (Table 2). Prospective cohort trials of sufficient size and follow-up that have adequately controlled for confounding, and selective 
follow-up is sufficiently excluded, obtained a level of evidence A2, while cohort studies not meeting these criteria or case-control studies obtained a level of evidence B. Non-controlled trials and expert opinions obtained a level of evidence $C$ and $D$, respectively.

\section{Strength of Conclusion}

Subsequently, the strength of conclusion (ranging from 1 to 4) was calculated for each cluster of studies reflecting one outcome parameter (Table 3), and was placed between brackets in the results section. Strength of conclusion 1 was assigned for a study of level A1 or at least 2 independently conducted studies of level A2. Strength of conclusion 2 was given when at least 2 independently conducted studies of evidence level B or one trial of evidence level A2 was included in the cluster, and strength of conclusion 3 was assigned if one study of evidence level B or C was present. Strength of conclusion 4 was given in case of inconclusive or inconsistent results between various studies.

\section{Results}

\section{Study Selection}

In total, 477 studies were retrieved from the different databases. After the first screening, 26 studies were identified as potential eligible studies for inclusion. After the second screening, only 12 studies were retrieved that fulfilled all inclusion criteria. The selection process of relevant articles is presented in Fig. 1.

\section{Study Characteristics}

The characteristics of each study were extracted and presented in the evidence table (Table 4). Four studies reported on structural brain alterations in neck pain patients (47-50) using a different MRI protocol. One study reported on changes in BOLD-signal through task-related fMRI-imaging (51), another one reported on alterations in neurotransmission through PET imaging (52), and 6 studies analyzed perfusion and/or metabolism via PET or SPECT-imaging using radiopharmaceutical tracers (53-58). The average age (+/- SD) of the total patient and control sample, when available, was $36.41(+/-11.52)$ years and $33.0(+/-12.21)$ years, respectively. In total, $75 \%(n=179)$ of the study participants were female. Eleven studies reported on patients after a whiplash injury or patients with WAD. Both longitudinal $(48,49)$ and cross-sectional studies $(47,50,51,53-58)$ have been included, using time periods ranging from 14 days to more than one year after the whiplash trauma before performing the scanning protocol. The key findings of this systematic review are depicted in Figs. 2 and 3.

\section{Risk of Bias in Individual Studies}

Cross-sectional studies obtained a score ranging from $2 / 9(22 \%)$ to $8 / 9(89 \%)$ for risk of bias with a median score of $4.5 / 9(50 \%)$. The risk of bias in studies was mostly high due to a lack of representativeness of cases in 6 out of 10 studies (60\%) (54-58), indicating the lack of a random sample. In 6 cases $(60 \%)$, the

Table 2. Level of evidence, according to the 2005 classification system of the Dutch Institute for Healthcare Improvement CBO.

\begin{tabular}{|c|l||}
\hline \multicolumn{2}{|l||}{ Intervention } \\
\hline A1 & Systematic review of at least 2 independent from each other conducted studies of evidence level A2 \\
\hline A2 & Randomized double-blinded comparative clinical research of good quality and efficient size \\
\hline B & $\begin{array}{l}\text { Comparative research, but not with al characteristics as mentioned for A2. This includes also patient-control research and cohort } \\
\text { research. }\end{array}$ \\
\hline C & Not comparative research \\
\hline D & Opinion of experts \\
\hline
\end{tabular}

Table 3. Strength of Conclusion (modified table).

\begin{tabular}{|c|l||}
\hline \multicolumn{2}{|l||}{ Conclusion based on } \\
\hline 1 & Research of evidence level A1 or at least 2 independent conducted studies of evidence level A2 \\
\hline 2 & 1 research of evidence level A2 or at least 2 independent conducted studies of evidence level B \\
\hline 3 & 1 research of evidence level B or C \\
\hline 4 & Inconclusive or inconsistent results between various studies \\
\hline
\end{tabular}




\section{Potentially relevant citations identified: 477}

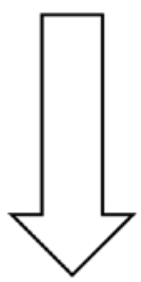

\section{Based on title and abstract evaluation, \\ citations excluded: 451 \\ Population: 390 \\ Intervention: 6 \\ Outcome: 37 \\ Design: 17 \\ Full text not available: 0}

Studies retrieved for more detailed evaluation: 26

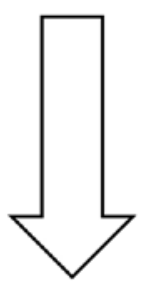

Based on full text evaluation, citations
excluded: 14
Population: 5
Intervention: 0
Outcome: 5
Design: 2
Full text not available: 2

Relevant studies included in qualitative synthesis: 12

Fig. 1. Study selection process.

researchers were not blinded to the patient's condition $(52-56,58)$, and 6 authors $(60 \%)$ did not clearly describe how the quality of the images was assessed $(47,50,53,55,56,58)$. However, only one study $(10 \%)$ did not provide a qualitative description of the cases included in their studies (56), and one study (10\%) did not provide a detailed brain imaging protocol (47). Cohort-studies, of which only 2 were eligible for this systematic review $(48,49)$, obtained a score of $3 / 11$ $(27 \%)$ and $6 / 11(54 \%)$. Both studies lost points on the representativeness of cases, selection of controls, new cases, quality control of images, and blinding the researchers for the patient's condition. In most cases $(96 \%)$, the 2 reviewers (RDP and IC) agreed. After a second review and a comparison of the differences, the reviewers reached a consensus for all items. The risk of bias in the individual studies is presented in Table 1.

\section{Synthesis of Results}

\section{Alterations in morphology}

Two studies have examined structural abnormalities shortly after the occurrence of a whiplash injury 
Is Traumatic and Non-traumatic Neck Pain Associated with Brain Alterations?

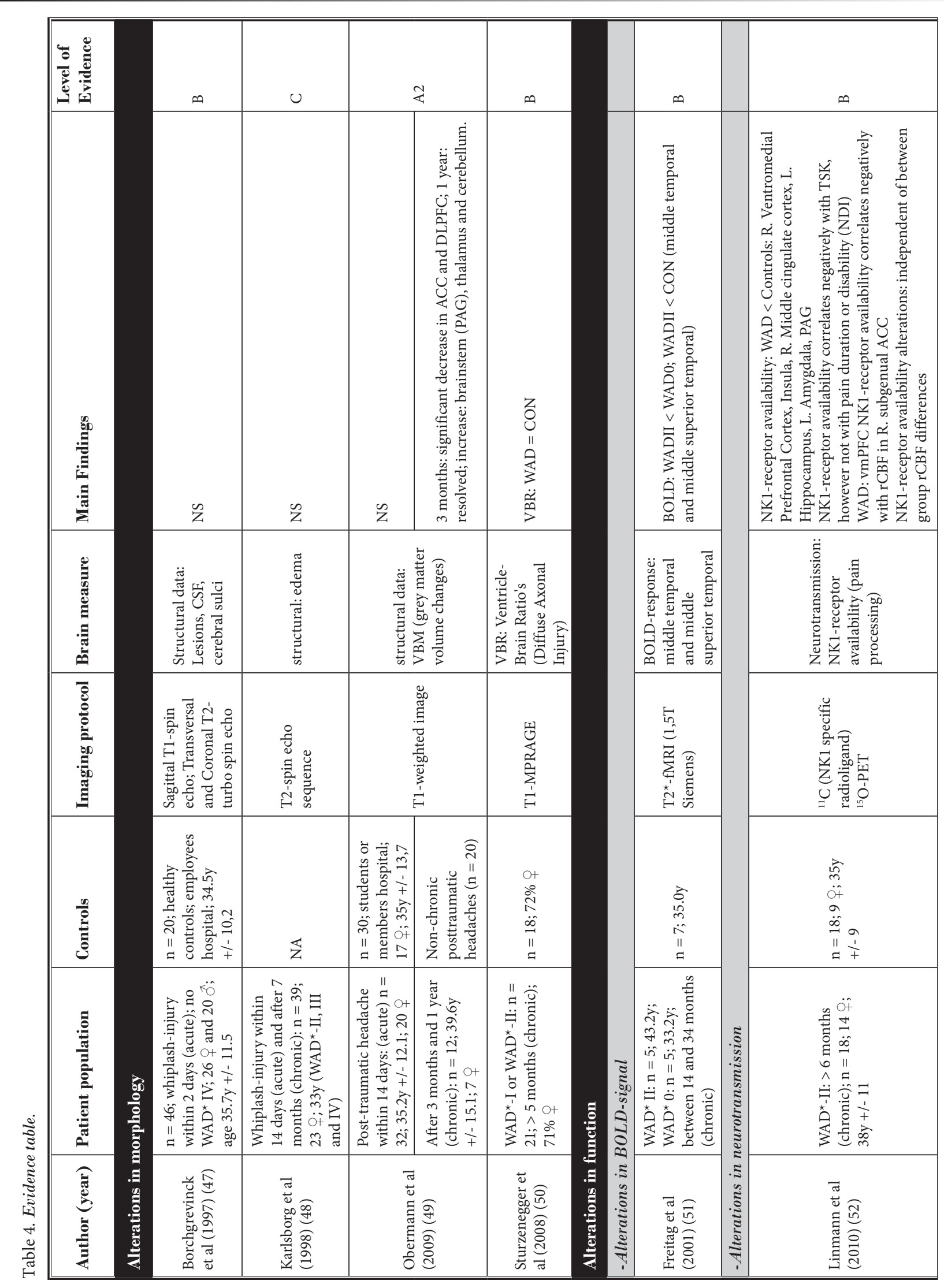


Pain Physician: May/June 2017: 20:245-260

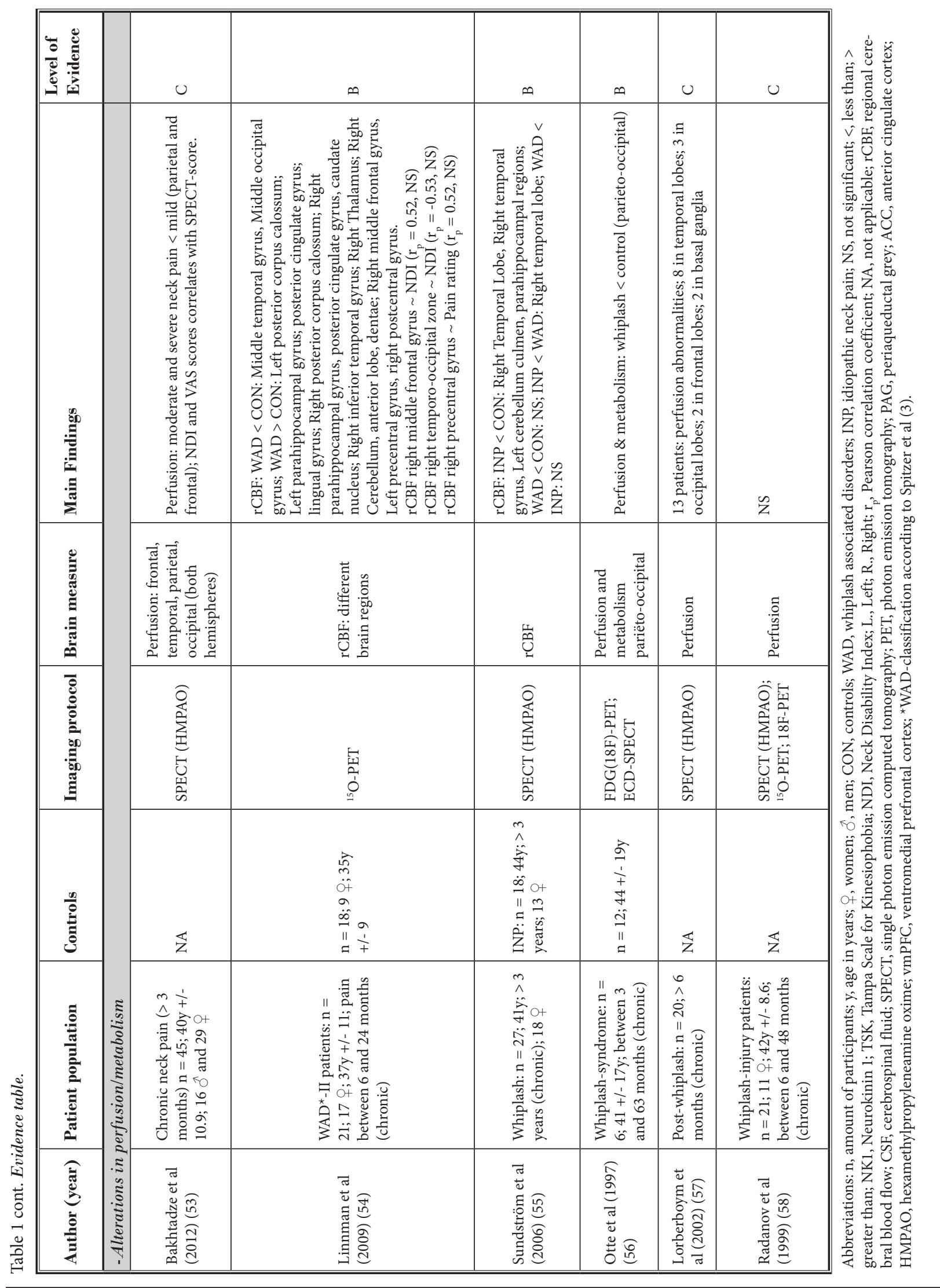



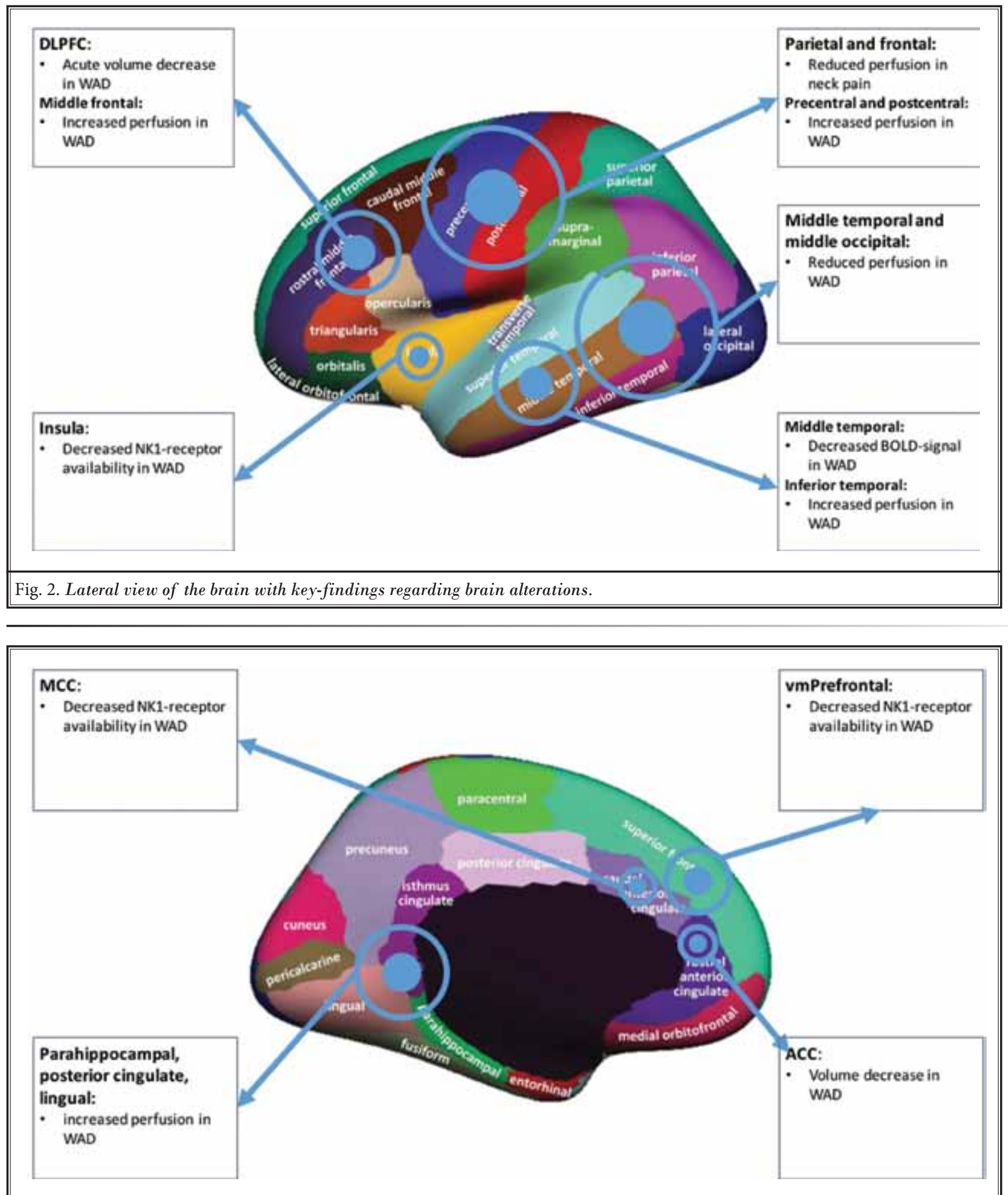

Fig. 3. Medial view of the brain with key-findings regarding brain alterations.

SOURCE: Figs. 2 and 3 are from An automated labeling system for subdividing the human cerebral cortex on MRI scans into gyral based regions of interest, Desikan et al (97). 
(within 2 to 14 days), but found no signs of edema in the acute whiplash group (47) nor signs of lesions when they compared patients after a whiplash injury with healthy controls (48). Another study analyzed the ventricle-brain ratio (VBR) - calculated as the ratio of the total ventricle volume divided by the brain volume and normalized afterwards by accounting for the average distance between the frontal and occipital poles of the entire study group (50). The authors found no difference in VBR between patients with chronic WAD and healthy controls (50). A study that performed voxel-based morphometry in patients with post-traumatic headache and neck pain, found no structural brain alterations in the acute phase (49). However, decreased grey matter volume in the anterior cingulate cortex (ACC) and dorsolateral prefrontal cortex (DLPFC) was observed after 3 months. These grey matter changes did, however, resolve after one year coinciding with the cessation of the post-traumatic headache. In comparison, an increase in grey matter volume was observed in the periaqueductal grey matter (PAG), the thalamus, and cerebellum (49).

To conclude, moderate evidence exists for a lack of structural pathological brain abnormalities, such as edema or lesions in acute traumatic neck pain patients who have suffered from a whiplash injury (Strength of conclusion 2). Moderate evidence exists for grey matter volume alterations after a certain period of time in patients with post-traumatic headache (Strength of conclusion 2).

\section{Alterations in function (BOLD-signal)}

One study examined the BOLD-signal at visual areas (middle temporal and superior temporal) in patients with chronic WAD (51). Therefore, patients were exposed to a visual task in the scanner. Two fields of dots were presented to the patient by projection. One field was positioned in the left visual field, whereas the other field was presented in the right visual field. On both screens dots moved in random directions, and the patients had to fixate their eyes on a central spot. At random, coherent movement of dots was added to one of the screens resulting in a mixture of random and coherent motion, and the screen with this mixture had to be reported by the patients to the researchers. The authors demonstrated a lower BOLD-response during coherent motion perception in the symptomatic chronic WAD group compared to asymptomatic persons after a whiplash trauma and healthy controls.

In conclusion, some evidence exists for functional brain alterations in temporal regions in patients with chronic whiplash (Strength of conclusion 3).

\section{Alterations in function (neurotransmission)}

Only one study investigated functional brain alterations related to neurotransmission (52). Linnman et al (52) analyzed the Neurokinin 1 (NK1)-receptors, a receptor mostly mediated by the neuropeptide Substance $P(S P)$, which allows the regulation of affective behavior, emesis, and nociception (59). In their study a decrease of NK1-receptor availability was found in chronic WAD patients (52), which was observed in the insula, right middle cingulate cortex, left hippocampus, left amygdala, and the PAG, but most distinct in the right ventromedial prefrontal cortex (vmPFC). Furthermore, these changes were negatively correlated with the scores on the self-reported Tampa Scale for Kinesiophobia (TSK) Questionnaire. Also, vmPFC NK1-receptor availability was negatively correlated with regional cerebral blood flow ( $\mathrm{rCBF}$ ) in the right subgenual ACC in chronic WAD patients.

In conclusion, some evidence exists for a decrease in NK1-receptor availability in pain processing brain regions in patients with chronic WAD (Strength of conclusion 3). Furthermore, there is some evidence that decreased vmPFC NK1-receptor availability is negatively correlated with $\mathrm{rCBF}$ in the ACC in chronic WAD patients (Strength of conclusion 3).

\section{Alterations in perfusion/metabolism}

In total, 6 studies examined alterations in perfusion/metabolism of the brain through SPECT or PET imaging (53-58). Only one study found no indication for changes of brain perfusion (58). Sundström et al (55) demonstrated that patients with chronic idiopathic neck pain showed a decreased rCBF pattern compared to healthy controls, which was most obvious in the parahippocampal and temporal regions and the cerebellum. In contrast, no such alterations could be observed in patients suffering from chronic WAD compared with healthy controls (60). Linnman et al (54) analyzed the rCBF in patients with chronic WAD and found alterations in the left parahippocampal gyrus, lingual gyrus, and posterior cingulate gyrus. In addition, alterations were also evident in the right parahippocampal gyrus, caudate nucleus, pulvinar nucleus of the thalamus, and posterior cingulate gyrus. Perfusion appeared higher in 
these regions in patients with chronic WAD, whereas in regions around the temporo-occipital transition zone, a decreased perfusion was found in these patients (54). In a more recent study of Bakhtadze et al (53), a decreased perfusion of the parietal and frontal region was found in patients who suffered from moderate to severe chronic idiopathic neck pain symptoms when compared to patients with only mild symptoms. Moreover, perfusion (SPECT-score) correlated with the amount of pain-related disability (utilizing the Neck Disability Index) (53). Two studies analyzed the perfusion in chronic WAD after a period of 6 months, and both found abnormalities in the patient group $(55,57)$. One study found predominantly decreased perfusion rates in patients with chronic idiopathic neck pain compared to healthy controls in the right temporal gyrus and left cerebellum culmen (55). However, no changes were found in the observation of perfusion in chronic WAD (55). This is in contrast with the study of Lorberboym et al (57), who found perfusion abnormalities in 13 of the 20 included patients with chronic WAD. However, these abnormalities were not equal for all patients. In 8 patients, perfusion abnormalities were observed in the temporal lobes, in 3 patients in the occipital lobes, in 2 patients in the frontal lobes, and another 2 patients showed perfusion abnormalities in the basal ganglia (57).

To conclude, moderate evidence exists for alterations in brain perfusion and metabolism in chronic neck pain patients (Strength of conclusion 2); however, the nature and location of these alterations is not entirely obvious. In addition, 2 studies found contradictory results for the association between clinical parameters and brain perfusion/metabolism in patients with chronic neck pain, resulting in strength of conclusion 4.

\section{Discussion}

This systematic review aimed to summarize and critically appraise the current state of the literature concerning brain alterations in patients with WAD and INP. Researchers have attempted to construct a solid hypothesis regarding the appearance of brain alterations in chronic pain and its associated symptoms $(61,62)$. However, only some to moderate evidence exists for empirical findings of structural and functional brain alterations in patients with chronic neck pain.

Some of the included studies scored poorly on the modified NOS, signifying the potential risk of bias included in the sample of studies discussed in this review. It is important that in future research a random sample of the study population and the healthy controls is included. New neuroimaging research indicates an important role of confounders in brain research, such as gender (63), age (64-68), BMI $(69,70)$, medication use $(71,72)$, and cognitive function (73). These confounders should be included by future neuroimaging studies into their statistical analysis to avoid biased estimates. About $75 \%$ of the included population were women, which is similar with current epidemiological data of (chronic) neck pain patients (1). In addition, most studies reported no statistical significant difference in age between the patient population and the included controls.

\section{Morphological Brain Alterations}

Although evidence for structural brain alterations in patients with chronic neck pain remains scarce, we can conclude that no structural brain abnormalities seem to be present in the acute phase after a whiplash trauma. Different authors observed nor signs of edema or lesions $(47,48)$, nor underlying structural differences between patients with chronic post-traumatic headache, acute post-traumatic headache, and healthy controls within 14 days after the accident, which makes morphometric predisposition in the affected patient group less likely (49). However, more longitudinal studies with a sufficiently large follow-up period are necessary to confirm these results and to determine a time frame for possible changes. After a 3-month period, decreased grey matter volume in the ACC and DLPFC was observed in whiplash patients who developed chronic headache. Yet, these changes were observed to resolve after one year in concurrence with the cessation of the headache. In contrast, an increased grey matter volume was observed in the PAG, thalamus, and cerebellum after one year in patients with chronic headache (49). These regions are known to be involved in pain sensation. The ACC and DLPFC are involved in the salience and affective-cognitive dimension of pain, and may play an important role in pain modulation, exerting top down inhibition (74-76). A disruption of the grey matter integrity of these regions might alter pain sensation. One study did identify the DLPFC as a site of major neurodegeneration in chronic pain patients (77), potentially leading to an increased pain sensation (78). In addition, increased grey matter volume of the PAG was demonstrated in patients with chronic WAD (49). The PAG is a brainstem structure that is part of the descending pain modulatory network and is crucially involved in pain inhibition or antinociception. These 
changes are furthermore in accordance with the current literature, where authors found primarily an increase of grey matter morphology in the brain stem (74). It has been suggested that the observed neuroplasticity (increase and decrease of grey matter morphology) might result from an aim to restore the balance between nociceptive and antinociceptive modulation (49). These changes in morphology seem to be very different across different pathologies and depend on timing (40). This diversity might correspond with the diversity of perfusion abnormalities in patients with chronic WAD found by Lorberboym et al (57), which are discussed in the section on functional brain alterations.

Sturzenegger et al (50) found no difference in VBR in patients suffering from chronic WAD, indicating their sample did not show any signs of diffuse axonal injury (DAI). They did not observe a correlation between VBR and any neuropsychological test (50). These results are somewhat surprising as diffuse axonal injuries have already been observed in patients with MTBI (79), who suffer from similar symptoms as patients with WAD. Also, Caeyenberghs et al $(80,81)$ have reported the presence of an association between postural control and visuomotor tracking, and a measure of DAI in patients with TBI. The use of Pearson correlations could contribute to the reason for not finding any associations between DAl and the patients' symptoms, as this measure only analyzes a linear relationship between 2 variables. Furthermore, the authors stated that the method they used to evaluate brain tissue loss may not be sensitive enough to detect very mild diffuse focal atrophy (50). A newer and potentially more sensitive technique for assessing white matter tracts is diffusion weighted imaging (DWI), a technique that indirectly analyzes the coherence of motion of protons $(82,83)$.

\section{Functional Brain Alterations}

One study examined alterations in neurotransmission by looking at the density of NK1-receptors in the brain (52), which are widely distributed throughout the brain with high density in the striatum, the amygdala, and the DLPFC (84). This receptor allows the modulation of pain via the neuropeptide SP, known to be elevated in patients with chronic WAD (85). Both, SP and NK1, have been implicated in locomotive activity (86) and in pain processing (87). Therefore, it is not surprising that the forebrain not only acts as a "top-down" pain inhibitor, but also modulates voluntary movement by altering the inhibition exerted by the basal ganglia on motor output, resulting in pain induced immobilization
(52). A decrease in NK1-receptor availability, which was most pronounced in the VmPFC of patients with chronic WAD (52), may provide forebrain modulation through its dense projections to the striatum-globus pallidus complex (88), which is supported by the observed negative correlation between kinesiophobia and NK1receptor availability (54). Consequently, high endogenous levels of SP could result in attenuation of NK1 function in the VmPFC, starting a negative vicious circle of increased avoidance (54). Furthermore, decreased grey matter volume in the forebrain ( $v m P F C$ ) in pain processing regions was already observed in patients with chronic complex regional pain syndrome together with reduced white matter integrity (89).

Alterations in the brain perfusion of patients with chronic idiopathic neck pain and chronic WAD have been observed by different studies (53-58). The diversity of results in brain perfusion and metabolism might be attributed to both methodological differences and technical issues. The use of different tracers results in different observations. Oxygen-15 (15-O) is for example often applied to determine blood flow, while fluorine-18 (18-F), which is injected as glucose, provides information on the brain's metabolism. Some authors state that blood flow provides more accurate information on brain function compared to glucose consumption, as it is more sensitive to neural activation (90). These differences make comparison between both methods rather difficult. The comparison between SPECT and PET imaging remains challenging due to differences in technical features, such as the use of different tracers and sensitivity, with PET being more sensitive to neural events (36). Thus, only cautious interpretations can be drawn from the comparison between studies that applied different imaging methods. The diversity in brain perfusion alterations within one technique could again support the hypothesis of individual adaptations to restore the equilibrium between nociceptive and antinociceptive modulation (57). Surprisingly, many brain areas were found to exhibit a higher amount of rCBF, which may reflect a compensation mechanism for regional brain atrophy (91). In addition, one study found differences between patients with chronic idiopathic neck pain and patients with WAD (55). These results might suggest that different mechanisms underlie the transition to a chronic or recurrent pain state in both patient groups, which is in accordance with other study areas (22).

Although the direction of brain perfusion alterations is still unclear, most authors do agree on the pres- 
ence of perfusion alterations in patients with chronic neck pain. Furthermore, research suggests the presence of an association between the patient's self-reported disability (NDI and pain ratings) and cerebral perfusion $(53,54)$. Therefore, future research should rather focus on the association between brain alterations and the severity of self-reported disability (53), although differences in brain activation between patients with chronic WAD and chronic INP were already observed (60). Only one study did not find any associations between brain activation and neuropsychological tests of divided attention and working memory (58).

\section{Limitations and Strengths}

When interpreting the results, the following study limitations have to be taken into consideration. Firstly, only 12 articles were included in this review, which allows only cautious conclusions to be drawn. Secondly, the diversity in brain imaging techniques hampers the possibility of comparison, as we are aware of the different technical features in every technique, which potentially affect the observed outcome. Thirdly, authors tend to use different "brain atlases" for analyzing and describing their results. Some analyzed a global region, such as the temporal lobe, while others investigated specific parts of a certain lobe, such as the vmPFC. Many researchers have tried to address a certain function to the brain's anatomy and have attributed a specific function to certain brain areas $(92,93)$. Lastly, many studies did suffer from certain risks of bias, which could affect their results, and this makes it impossible to draw firm conclusions from the current literature.

However, several strengths of this systematic review can be outlined. Firstly, the present systematic review is innovative and is valuable to steer future brain research. Secondly, the methods used for screening and scoring were completed by 2 independent blinded researchers. And last, the NOS checklist was modified by adding 2 MRI-related scoring items specifically developed for the topic of the current systematic review. Consequently, the methodological quality of the MRI articles could be evaluated more thoroughly giving a more accurate view on the MRI data acquisition, processing, and quality control.

\section{Recommendations for Further Research}

Future studies should certainly try to avoid bias, and should consider the mentioned considerations regarding the inclusion of confounding factors. Furthermore, more longitudinal research could allow exploring the causal relationship between brain imaging results and the development and maintenance of persistent neck pain. In addition, research on disease-specific neck pain could reveal different neuroplastic brain changes when compared to non-specific neck pain.

To date, the imaging techniques used in studies that have assessed the brain in a population of neck pain patients are outdated. Recently, new morphological brain analysis tools were developed with new features allowing a more detailed assessment of the human brain. Surface-based morphometry (SBM) has yielded better results in terms of specificity compared to voxel-based morphometry $(31,42)$. One reason is the ability of SBM to assess not only volumetric measurements, but also measures of cortical thickness and cortical surface area. Alterations in thickness and area cause different clinical effects, and non-uniformity of these changes has been shown in the brain in aging (65). DWI has also yielded better results in terms of analyzing the microstructural organization of white matter bundles (82). Besides improvement in morphological imaging, functional brain imaging has also evolved. One new popular method, defined as "resting state fMRI" could give more insight into the functional organization of the brain during rest (94). Recently, a new theoretical framework has risen which addresses the influence of alterations in the aggregation of different functional components of the brain and its influence on pain (4). Lastly, alterations in the network of the brain have been given attention in different conditions, including chronic pain conditions (95). Assessing the morphological and functional brain network could allow assessment of the macroscopic organization of this complex organ (96).

\section{Conclusion}

Some to moderate evidence exists for both structural and/or functional brain alterations in patients with chronic INP or WAD. In the acute phase, no structural alterations were found, but if symptoms persisted, changes in different brain areas were demonstrated. Although, most authors agree that brain alterations are present in both patients with chronic WAD and patients with chronic INP, there is currently more evidence for brain alterations in chronic whiplash patients, and different underlying mechanisms might be present in both pathologies. Moreover, brain alterations observed in chronic neck pain patients are very diverse, indicating multiple mechanisms are responsible for the brain's neuroplasticity associated with the presence of pain. Pain and disability seems to be furthermore correlated 
with the observed brain alterations. Based on our results, morphological and functional brain alterations should be further investigated in patients with chronic WAD and chronic INP via more sophisticated and sensi- tive techniques.

\section{References}

1. Hogg-Johnson S, van der Velde G, Carroll LJ, Holm LW, Cassidy JD, Guzman J, Cote P, Haldeman S, Ammendolia C, Carragee E, Hurwitz E, Nordin M, Peloso P. The burden and determinants of neck pain in the general population - Results of the Bone and Joint Decade 2000 - 2010 Task Force on Neck Pain and its Associated Disorders. Eur Spine J 2008; 17:S39-S51.

2. Carroll LJ, Hogg-Johnson S, van der Velde G, Haldeman S, Holm LW, Carragee E), Hurwitz EL, Côté P, Nordin M, Peloso PM, Guzman J, Cassidy JD. Course and prognostic factors for neck pain in the general population: Results of the Bone and Joint Decade 2000 - 2010 Task Force on Neck Pain and Its Associated Disorders. Eur Spine ] 2008; 17:75-82.

3. Spitzer WO, Skovron ML, Salmi LR, Cassidy JD, Duranceau J, Suissa S, Zeiss E. Scientific monograph of the Quebec Task Force on Whiplash-Associated Disorders: Redefining "whiplash" and its management. Spine 1995; 20:1s-73s.

4. Davis CG. Mechanisms of chronic pain from whiplash injury. J Forensic Leg Med 2013; 20:74-85.

5. Sterling M, Jull G, Kenardy J. Physical and psychological factors maintain long-term predictive capacity postwhiplash injury. Pain 2006; 122:102-108.

6. Rebbeck T, Sindhusake D, Cameron I, Rubin G, Feyer A, Walsh J, Gold M, Schofield W. A prospective cohort study of health outcomes following whiplash associated disorders in an Australian population. Inj Prev 2006; 12:93-98.

7. Treleaven J. Sensorimotor disturbances in neck disorders affecting postural stability, head and eye movement control. Man Ther 2008; 13:2-11.

8. Baydal-Bertomeu JM, Page ÁF, BeldaLois JM, Garrido-Jaén D, Prat JM. Neck motion patterns in whiplash-associated disorders: Quantifying variability and spontaneity of movement. Clinical Biomechanics 2011; 26:29-34.

9. Dall'Alba PT, Sterling MM, Treleaven JM, Edwards SL, Jull GA. Cervical range of motion discriminates between as- ymptomatic persons and those with whiplash. Spine 2001; 26:2090-2094.

10. Grip H, Sundelin G, Gerdle B, Karlsson JS. Variations in the axis of motion during head repositioning - A comparison of subjects with whiplash-associated disorders or non-specific neck pain and healthy controls. Clin Biomech 2007; 22:865-873.

11. Sjölander P, Michaelson P, Jaric S, Djupsjöbacka M. Sensorimotor disturbances in chronic neck pain — range of motion, peak velocity, smoothness of movement, and repositioning acuity. Man Ther 2008; 13:122-131.

12. Woodhouse A, Stavdahl $\varnothing$, Vasseljen O. Irregular head movement patterns in whiplash patients during a trajectory task. Exp Brain Res 2010; 201:261-270.

13. Meisingset I, Woodhouse A, Stensdotter A-K, Stavdahl $\varnothing$, Lorås H, Gismervik $\mathrm{S}$, Andresen $\mathrm{H}$, Austreim K, Vasseljen O. Evidence for a general stiffening motor control pattern in neck pain: A cross sectional study. BMC Musculoskelet Disord 2015; 16:56.

14. Van Oosterwijck J, Nijs J, Meeus M, Paul $L$. Evidence for central sensitization in chronic whiplash: A systematic literature review. Eur J Pain 2013; 17:299-312.

15. Stone AM, Vicenzino B, Lim EC, Sterling M. Measures of central hyperexcitability in chronic whiplash associated disorder - a systematic review and meta-analysis. Man Ther 2013; 18:111-117.

16. Coppieters I, Ickmans K, Cagnie B, Nijs J, De Pauw R, Noten S, Meeus M. Cognitive performance is related to central sensitization and health-related quality of life in patients with chronic whiplashassociated disorders and fibromyalgia. Pain Physician 2015; 18:E389-E401.

17. Treleaven J, Jull G, Sterling M. Dizziness and unsteadiness following whiplash injury: Characteristic features and relationship with cervical joint position error. J Rehabil Med 2003; 35:36-43.

18. Rubin AM, Woolley SM, Dailey VM, Goebel JA. Postural stability following mild head or whiplash injuries. Otol Neurotol 1995; 16:216-221.
19. Kessels RP, Aleman A, Verhagen WI, Van Luijtelaar EL. Cognitive functioning after whiplash injury: A meta-analysis. J Int Neuropsychol Soc 2000; 6:271-278.

20. Borghouts JA, Koes BW, Bouter LM. The clinical course and prognostic factors of non-specific neck pain: A systematic review. Pain 1998; 77:1-13.

21. Scott D, Jull G, Sterling M. Widespread sensory hypersensitivity is a feature of chronic whiplash-associated disorder but not chronic idiopathic neck pain. Clin J Pain 2005; 21:175-181.

22. De Pauw R, Coppieters I, Kregel J, De Meulemeester K, Danneels L, Cagnie $B$. Does muscle morphology change in chronic neck pain patients? - A systematic review. Man Ther 2016; 22:42-49.

23. Woodhouse A, Vasseljen O. Altered motor control patterns in whiplash and chronic neck pain. BMC Musculoskelet Disord 2008; 9:90.

24. Hodges PW, Tucker K. Moving differently in pain: A new theory to explain the adaptation to pain. Pain 2011; 152:S90-S98.

25. Heikkilä HV, Wenngren B-I. Cervicocephalic kinesthetic sensibility, active range of cervical motion, and oculomotor function in patients with whiplash injury. Arch Phys Med Rehabil 1998; 79:1089-1094.

26. Wenngren $\mathrm{BI}$, Pettersson K, Löwenhielm G, Hildingsson C. Eye motility and auditory brainstem response dysfunction after whiplash injury. Acta Otolaryngol 2002; 122:276-283.

27. Mosimann UP, Müri RM, Felblinger J, Radanov BP. Saccadic eye movement disturbances in whiplash patients with persistent complaints. Brain 2000; 123:828-835.

28. Apkarian AV, Bushnell MC, Treede RD, Zubieta JK. Human brain mechanisms of pain perception and regulation in health and disease. Eur J Pain 2005; 9:463-484.

29. Apkarian AV, Baliki MN, Geha PY. Towards a theory of chronic pain. Prog Neurobiol 2009; 87:81-97.

30. Alexander MP. Mild traumatic brain in- 
jury: Pathophysiology, natural history, and clinical management. Neurol 1995: 45:1253-1260.

31. Fischl B. FreeSurfer. Neuroimage 2012; 62:774-781.

32. Ashburner J, Friston KJ. Voxel-based morphometry - the methods. Neuroimage 2000; 11:805-821.

33. Alexander AL, Lee JE, Lazar M, Field AS. Diffusion tensor imaging of the brain. Neurotherapeutics 2007; 4:316-329.

34. Tournier JD, Mori S, Leemans A. Diffusion tensor imaging and beyond. Magn Reson Med 2011; 65:1532-1556.

35. Jeurissen B, Leemans A, Tournier JD, Jones DK, Sijbers J. Investigating the prevalence of complex fiber configurations in white matter tissue with diffusion magnetic resonance imaging. Hum Brain Mapp 2013; 34:2747-2766.

36. Rahmim A, Zaidi H. PET versus SPECT: Strengths, limitations and challenges. Nucl Med Commun 2008; 29:193-207.

37. Warwick JM. Imaging of brain function using SPECT. Metab Brain Dis 2004; 19:113-123.

38. Dienel GA, Hertz L. Glucose and lactate metabolism during brain activation. J Neurosci Res 2001; 66:824-838.

39. Amaro E, Barker GJ. Study design in fMRI: Basic principles. Brain Cogn 2006; 60:220-232.

40. Baliki MN, Schnitzer TJ, Bauer WR, Apkarian AV. Brain morphological signatures for chronic pain. PLOS ONE 2011; 6:e26010-e26010.

41. Schmidt-Wilcke T, Ganssbauer S, Neuner T, Bogdahn U, May A. Subtle grey matter changes between migraine patients and healthy controls. Cephalalgia 2008; 28:1-4.

42. Clarkson MJ, Cardoso MJ, Ridgway GR, Modat M, Leung KK, Rohrer JD, Fox NC, Ourselin S. A comparison of voxel and surface based cortical thickness estimation methods. Neuroimage 2011; 57:856-865

43. Moher D, Liberati A, Tetzlaff J, Altman DG. Preferred reporting items for systematic reviews and meta-analyses: The PRISMA statement. Int ] Surg 2010; 8:336-341.

44. Moher D, Shamseer L, Clarke M, Ghersi D, Liberati A, Petticrew M, Shekelle $P$, Stewart LA. Preferred reporting items for systematic review and meta-analysis protocols (PRISMA-P) 2015 statement. Syst Rev 2015; 4:1.

45. Wells G SB, O'Connell D, Peterson J,
Welch V, Losos M, Tugwell P. The Newcastle-Ottawa Scale (NOS) for Assessing the Quality of Nonrandomised Studies in Meta-Analyses. 2014 [cited Accessed in September, 2015.]. Available from: www.ohri.ca/programs/clinical_epidemiology/ oxford.asp.

46. Zeng X, Zhang Y, Kwong JS, Zhang C, Li $S$, Sun F, Niu Y, Du L. The methodological quality assessment tools for preclinical and clinical studies, systematic review and meta-analysis, and clinical practice guideline: A systematic review. J Evid Based Med 2015; 8:2-10.

47. Borchgrevink G, Smevik O, Haave I, Haraldseth O, Nordby A, Lereim I. MRI of cerebrum and cervical columna within two days after whiplash neck sprain injury. Injury 1997; 28:331-335.

48. Karlsborg M, Smed A, Jespersen HF, Stephensen SL, Cortsen ME, Jennum PJ, Herning GM, Korfitsen E, Werdelin LM. Whiplash injury syndrome. A prospective study of 39 patients with whiplash injury]. Ugeskr Laeger 1998; 160:6211-6215

49. Obermann M, Nebel K, Schumann C, Holle D, Gizewski ER, Maschke M, Goadsby PJ, Diener HC, Katsarava Z. Gray matter changes related to chronic posttraumatic headache. Neurol 2009; 73:978-983.

50. Sturzenegger M, Radanov BP, Winter P, Simko M, Farra AD, Di Stefano G. MRIbased brain volumetry in chronic whiplash patients: No evidence for traumatic brain injury. Acta Neurol Scand 2008; 117:49-54

51. Freitag P, Greenlee MW, Wachter K, Ettlin TM, Radue EW. fMRI response during visual motion stimulation in patients with late whiplash syndrome. Neurorehabil Neural Repair 2001; 15:31-37.

52. Linnman C, Appel L, Furmark T, Soderlund A, Gordh T, Langstrom B, Fredrikson $M$. Ventromedial prefrontal neurokinin 1 receptor availability is reduced in chronic pain. Pain 2010; 149:64-70.

53. Bakhtadze MA, Vernon H, Karalkin AV, Pasha SP, Tomashevskiy IO, Soave D. Cerebral perfusion in patients with chronic neck and upper back pain: Preliminary observations. J Manipulative Physiol Ther 2012; 35:76-85

54. Linnman C, Appel L, Soderlund A, Frans $\mathrm{O}$, Engler H, Furmark T, Gordh T, Langstrom B, Fredrikson M. Chronic whiplash symptoms are related to altered regional cerebral blood flow in the resting state. Eur J Pain 2009; 13:65-70.
55. Sundstrom T, Guez M, Hildingsson C, Toolanen G, Nyberg L, Riklund K. Altered cerebral blood flow in chronic neck pain patients but not in whiplash patients: A 99mTc-HMPAO rCBF study. Eur Spine J 2006; 15:1189-1195.

56. Otte A, Mueller-Brand J, Nitzsche EU, Wachter K, Ettlin TM. Functional brain imaging in 200 patients after whiplash injury. J Nucl Med 1997; 38:1002.

57. Lorberboym M, Gilad R, Gorin V, Sadeh M, Lampl Y. Late whiplash syndrome: Correlation of brain SPECT with neuropsychological tests and $\mathrm{P}_{300}$ event-related potential. J Trauma 2002; 52:521-526.

58. Radanov BP, Bicik I, Dvorak J, Antinnes J, von Schulthess GK, Buck A. Relation between neuropsychological and neuroimaging findings in patients with late whiplash syndrome. J Neurol Neurosurg Psychiatry 1999; 66:485-489.

59. Mantyh PW. Neurobiology of substance $P$ and the NKı receptor. J Clin Psychiatry 2001; 63:6-10.

6o. Sundstrom T, Guez M, Hildingsson C, Toolanen G, Nyberg L, Riklund K. Altered cerebral blood flow in chronic neck pain patients but not in whiplash patients: A Tc-99m-HMPAO rCBF study. Eur Spine J 2006; 15:1189-1195.

61. Schmidt-Wilcke T. Neuroimaging of chronic pain. Best Pract Res Clin Rheumatol 2015; 29:29-41.

62. Baliki MN, Apkarian AV. Nociception, pain, negative moods, and behavior selection. Neuron 2015; 87:474-491.

63. Passe TJ, Rajagopalan P, Tupler LA, Byrum CE, Macfall JR, Krishnan KRR. Age and sex effects on brain morphology. Prog Neuropsychopharmacol Biol Psychiatry 1997; 21:1231-1237.

64. Lemaitre H, Goldman AL, Sambataro $\mathrm{F}$, Verchinski Ba, Meyer-Lindenberg A, Weinberger DR, Mattay VS. Normal age-related brain morphometric changes: Nonuniformity across cortical thickness, surface area and gray matter volume? Neurobiol Aging 2012; 33:e611-e617, e619.

65. Grady CL, Springer MV, Hongwanishkul D, Mclntosh AR, Winocur G. Agerelated changes in brain activity across the adult lifespan. J Cogn Neurosci 2006; 18:227-241.

66. Meunier D, Achard S, Morcom A, Bullmore E. Age-related changes in modular organization of human brain functional networks. Neuroimage 2009; 44:715-723.

67. Burke SN, Barnes CA. Neural plasticity 
in the ageing brain. Nat Rev Neurosci 2006; 7:30-40.

68. Ceko M, Bushnell MC, Fitzcharles MA, Schweinhardt P. Fibromyalgia interacts with age to change the brain. Neuroimage Clin 2013; 3:249-260.

69. Pannacciulli N, Del Parigi A, Chen K, Le DSN, Reiman EM, Tataranni PA. Brain abnormalities in human obesity: A voxel-based morphometric study. Neuroimage 2006; 31:1419-1425.

70. Raji CA, Ho AJ, Parikshak NN, Becker JT, Lopez OL, Kuller LH, Hua X, Leow AD, Toga AW, Thompson PM. Brain structure and obesity. Hum Brain Mapp 2010; 31:353-364.

71. Walther K, Bendlin B, Glisky E, Trouard T, Lisse J, Posever J, Ryan L. Anti-inflammatory drugs reduce age-related decreases in brain volume in cognitively normal older adults. Neurobiol Aging 2011; 32:497-505.

72. Younger JW, Chu LF, D'Arcy NT, Trott KE, Jastrzab LE, Mackey SC. Prescription opioid analgesics rapidly change the human brain. Pain 2011; 152:1803-1810.

73. Bendlin BB, Fitzgerald $M E$, Ries $M L$, Xu G, Kastman EK, Thiel BW, Rowley HA, Lazar M, Alexander AL, Johnson SC. White matter in aging and cognition: $A$ cross-sectional study of microstructure in adults aged eighteen to eighty-three. Dev Neuropsychol 2010; 35:257-277.

74. Davis KD, Moayedi M. Central mechanisms of pain revealed through functional and structural MRI. J Neuroimmune Pharmacol 2013; 8:518-534.

75. Baliki MN, Apkarian AV. Nociception, pain, negative moods, and behavior selection. Neuron 2015; 87:474-491.

76. Vachon-Presseau E, Centeno MV, Ren W, Berger SE, Tétreault P, Ghantous M, Bario A, Farmer M, Baliki MN, Schnitzer TJ, Apkarian AV. The emotional brain as a predictor and amplifier of chronic pain. Dent Res J 2016; 95:605-612.

77. Apkarian AV, Sosa Y, Sonty S, Levy RM, Harden RN, Parrish TB, Gitelman DR. Chronic back pain is associated with decreased prefrontal and thalamic gray matter density. J Neurosci 2004; 24:10410-10415.
78. Lorenz J, Minoshima S, Casey KL. Keeping pain out of mind: The role of the dorsolateral prefrontal cortex in pain modulation. Brain 2003; 126:1079-1091.

79. Inglese M, Makani S, Johnson G, Cohen BA, Silver JA, Gonen O, Grossman RI. Diffuse axonal injury in mild traumatic brain injury: A diffusion tensor imaging study. J Neurosurg 2005; 103:298-303.

8o. Caeyenberghs K, Leemans A, Geurts M, Taymans T, Linden CV, Smits-Engelsman B, Sunaert S, Swinnen SP. Brainbehavior relationships in young traumatic brain injury patients: DTI metrics are highly correlated with postural control. Hum Brain Mapp 2010; 31:992-1002.

81. Caeyenberghs K, Leemans A, Geurts $M$, Taymans T, Vander Linden C, SmitsEngelsman B, Sunaert S, Swinnen SP. Brain-behavior relationships in young traumatic brain injury patients: Fractional anisotropy measures are highly correlated with dynamic visuomotor tracking performance. Neuropsychologia 2010; 48:1472-1482.

82. Jones DK, Knösche TR, Turner R. White matter integrity, fiber count, and other fallacies: The do's and don'ts of diffusion MRI. Neuroimage 2013; 73:239-254.

83. Tournier J-D, Mori S, Leemans A. Diffusion tensor imaging and beyond. Magn Reson Med 2011; 65:1532-1556.

84. Hietala J, Nyman MJ, Eskola O, Laakso A, Grönroos T, Oikonen V, Bergman J, Haaparanta M, Forsback S, Marjamäki P. Visualization and quantification of neurokinin-1 $(\mathrm{NKI})$ receptors in the human brain. Mol Imaging Biol 2005; 7:262-272.

85. Alpar EK, Onuoha G, Killampalli VV, Waters R. Management of chronic pain in whiplash injury. Journal of Bone and Joint Surgery - British Volume 2002; 84:807-811.

86. Elliott PJ, Iversen SD. Behavioural effects of tachykinins and related peptides. Brain Res 1986; 381:68-76.

87. Woolf CJ, Salter MW. Neuronal plasticity: Increasing the gain in pain. Science 2000; 288:1765-1768.

88. Haber S, Kunishio K, Mizobuchi M, Lynd-Balta E. The orbital and medi- al prefrontal circuit through the primate basal ganglia. J Neurosci 1995; 15:4851-4867.

89. Geha PY, Baliki MN, Harden RN, Bauer WR, Parrish TB, Apkarian AV. The brain in chronic CRPS pain: Abnormal graywhite matter interactions in emotional and autonomic regions. Neuron 2008; 60:570-581.

90. Varvatsoulias G. The physiological processes underpinning PET and FMRI techniques with an emphasis on the temporal and spatial resolution of these methods. Psychological Thought 2013; 6:173-195.

91. Dickerson BC, Salat DH, Bates JF, Atiya $M$, Killiany RJ, Greve DN, Dale AM, Stern CE, Blacker D, Albert MS. Medial temporal lobe function and structure in mild cognitive impairment. Ann Neurol 2004; 56:27-35.

92. Cavanna AE, Trimble MR. The precuneus: A review of its functional anatomy and behavioural correlates. Brain 2006; 129:564-583.

93. Alvarez JA, Emory E. Executive function and the frontal lobes: A meta-analytic review. Neuropsychol Rev 2006; 16:17-42.

94. van den Heuvel MP, Hulshoff Pol HE. Exploring the brain network: A review on resting-state fMRI functional connectivity. Eur Neuropsychopharmacol 2010; 20:519-534.

95. Crossley NA, Mechelli A, Scott J, Carletti F, Fox PT, McGuire P, Bullmore ET. The hubs of the human connectome are generally implicated in the anatomy of brain disorders. Brain 2014; 137:2382-2395.

96. Rubinov M, Sporns O. Complex network measures of brain connectivity: Uses and interpretations. Neuroimage 2010; 52:1059-1069

97. Desikan RS, Florent S, Fischl B, Quinn BT, Dickerson BC, Blacker D, Buckner RL, Dale AM, Maguire RM, Hyman BT, Albert MS, Killianya RJ. An automated labeling system for subdividing the human cerebral cortex on MRI scans into gyral based regions of interest. Neuroimage 2006; 31:968-980. 